\title{
Power Saving Mechanism for Ad-Hoc Network using 3G Fast Dormancy Technology
}

\author{
D. Helen ${ }^{1 *}$ and D. Arivazhagan ${ }^{2}$ \\ Department of Information Technology, AMET University, Chennai, India; \\ helensaran15@gmail.com, arivazhagan@hotmail.com
}

\begin{abstract}
The 3G devices known for higher energy consumption. The energy demand can be minimized by avoiding unnecessary listening between the nodes using fast dormancy. The fast dormancy mechanism has been implemented in ad-hoc network. Thus the channel access done by introducing Ad-hoc Traffic Indication Window(ATIM) in ad-hoc network. The ATIM window size is defined in an optimal way. According to the ATIM window behavior the node may enter into awake / sleep state. Making the node in sleep state avoid the nodes overhearing in the network traffic as a result node failure will be avoided and network topology may maintain. The stimulation has been done with 100 nodes based on nodes throughput, energy and delay. The result shows long lived nodes in the network. Thus, the network structure has been maintained by saving nodes energy by implementing fast dormancy. The fast dormancy release the ad-hoc network channel when they no longer needed. The $90 \%$ of nodes alive active to maintain network topology.
\end{abstract}

Keywords: Channel, Energy, Fast Dormancy, Transmission

\section{Introduction}

The 3G technology supports the global roaming which allow the users to use the same number and device all over the world. India supports UMTS 2100 in 3G. The $3 \mathrm{G}$ technology is combined with Code Division Multiple Access (CDMA 2000), Time-division Synchronous Codedivision Multiple Access (TD-SCDMA) and Wideband Code Division Multiple Access (W-CDMA (UMTS)) technology. The data transfer rate is $3 \mathrm{Mbps}$ (spent 15 seconds to download a 3 minute MP3 song) for $3 \mathrm{G}$ mobile phones. The $3 \mathrm{G}$ technology was implied in the network protocol stack. Example, the $3 \mathrm{G}$ technology was mostly adopted by Samsung phones. 3G network use wide radio spectrum for faster communication and larger network capacity ${ }^{1}$.The $3 \mathrm{G}$ phones are regularly connected to the internet wherever the mobile network service exists ${ }^{2}$. The $3 \mathrm{G}$ technology also requires more power for communication resulting in frequent recharge. The 3GPP use fast dormancy method to save power by releasing the connection when no traffic exists for mobile communication.

Thus, the power management is one of the main inspiring issues in $3 \mathrm{G}$ mobile phones. The $3 \mathrm{G}$ technology was employed in smartphones and tablets for high speed data communication. The $3 \mathrm{G}$ mobile spends major amounts of energy during communication. Example, Samsung Nexus S use the battery power " 6 hours 40 minutes" for communication ${ }^{3}$. The existing research ${ }^{3}$ says, $60 \%$ of the power used by $3 \mathrm{G}$ interfaces during no transmission and reception. The significance is energy wasted due to overhearing nodes in the network.

The proposed paper provides the solution to save energy in $3 G$ when there is no communication. The 3G release 7 introduced the fast dormancy method for mobile device capable of disconnecting the communica-

${ }^{*}$ Author for correspondence 
tion when there is no traffic ${ }^{4}$. But there is no clear idea when to release the channel and when to activate it. In Europe, Nokia Siemens has deployed their network based on fast democracy in 3GPP Release ${ }^{8}$. The iPhone is running on iOS4.2 in the network and it supports fast dormancy ${ }^{5}$ but the mechanism is not clear. Presently there is no technique to demonstrate when the device need to initiate the fast dormancy ${ }^{6}$. There is a significance to use fast dormancy in MANET due to dynamic topology alteration ${ }^{2}$. The proposed paper discuss with flagging technique along with the AITM window to determine for the node to go for active state or sleep state.

\section{Fast Dormancy}

Recently, Nokia and Qualcom designed a technology called "fast dormancy" for battery saving ${ }^{5}$. The 3GPP release 8 introduced the fast dormancy for smartphone to automatically release the communication in an abnormal situation and goes to idle mode. Generally, the fast dormancy uses the Dynamic control channel (DCH ) for large volume of data and it use high power for consumption. During small volume of data transmission it uses the Forward Access Channel (FACH) for fast communication. The network change to Paging Channel (PCH) mode when there is no communication but needs to maintain the connection. The $3 \mathrm{G}$ network supports the fast dormancy method for saving the node power in the network. To enable/disable fast dormancy in smart phone type * ${ }^{* 900 \# ~ . ~ E x a m p l e: ~ T-M o b i l e ~ h a s ~ e v o l v e d ~ w i t h ~ f a s t ~}$ dormancy.

\section{Problem Definition}

The 3GPP proposed a feature called "fast dormancy" which allows to release the channel itself when there is no communication. Implementing fast dormancy in an adhoc network is a difficult task because of frequent node movement in the network topology. The network traffic may also change instantly. Standard committee suggests that the device should utilize the application layer knowledge for transmission. The proposed technique allows to release the channel when there in no network traffic.

\section{Methodology}

\subsection{ATIM Window}

IEEE802.11 defines the Distributed Point Coordination Function(DCF) for power management ${ }^{7}$. The DCF uses the beacon signal between nodes for synchronization. The beacon interval used the fixed time interval called ATIM window. The ATIM window informs the destination node to receive the packet from source. After receiving the window, source and destination stay awake for communication then there is a need for channel to access. Always the ATIM window never performed good when they defined with the window size ${ }^{8}$. If the ATIM window is too small the pending may occur and waits for another ATIM window as a result overload occurs in the transmission. If the ATIM window is too large then the unnecessary traffic will occur.Figure 1 explains the ATIM window structure.

The paper use the optimal window size ${ }^{8}$ the following metrics are used in the rest of the paper,

ATIMmin: It define the minimum window size for the node. The ATIM min is fixed to 20s.

ATIMmax: It set the maximum window size for the node. The ATIM max is set to 60 s.

Channel Status (CS): The channel status is checked at end of each ATIM window. If the channel is in an awake status the CS value is reset the flag bit to 0.Therfore there

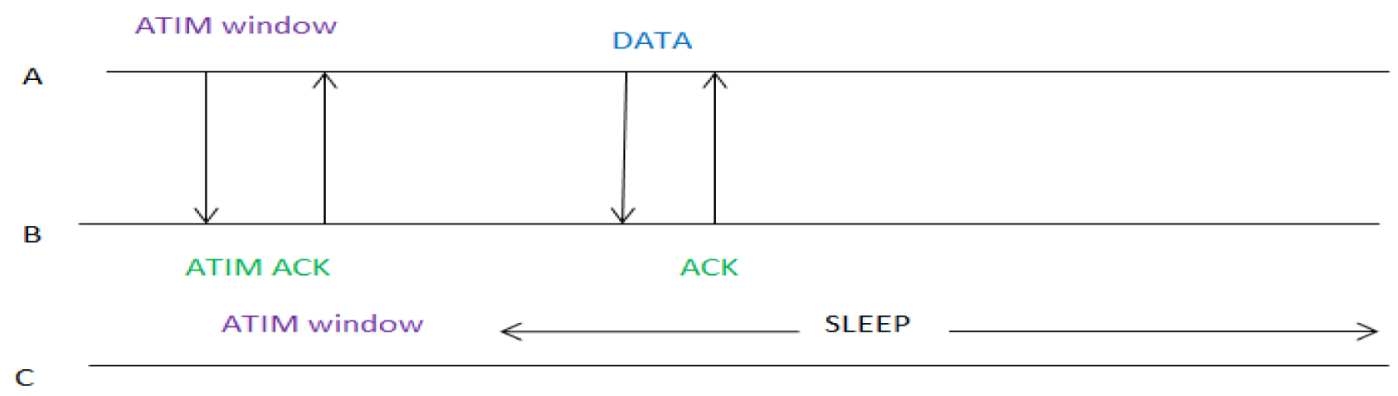

Figure 1. ATIM window structure. 
is not any necessary to release the channel. If the CS set the flag bit to 1 then the channel may release.

\subsection{Flag Setting}

The flag variable has one value used to check the condition .A flag variable, is defined in a simplest form, to check the condition until some condition is true, otherwise the variable value remains as per initialization where flag is false. The variable can use in control flow of function, allow to check for certain conditions while the function progresses. To demonstrate when to release and access the channel the proposed paper identify the flag value along with ATIM window. If the flag value is zero then there is a need for the channel. The flag bit is set to 1 then there is no need for connection release.

\subsection{Algorithm}

Input: $\mathrm{N}^{`}$ Number of Nodes
Output: Channel Allocation/ Release
Process:
1: ATIM window is set to ATIMmin;
2: flag :=0;
3: repeat
4: if ATIM window finished then
5: Node verify the CS
6: if (flag $=0$ ) and (ATIMmin $>$ AITMwin) then
7: Restart ATIM window uptoATIMmin
8: else
9: Allocate the channel for transmission
10: flag:= 1
11: end if
12: end if
13: until flag=1

\subsection{Proposed System}

Energy maintenance is one of main factor in ad-hoc network ${ }^{9}$. In proposed paper, node uses the ATIM window to transmit the packet. The ATIM window establish with regular beacon interval. The interval time is 120 s for per window. The node starts the communication during the window flag bit is set to 0 and ATIMmin value is equal to 20s. This is used to identify the traffic in the network. The node release the channel and goes to sleep state when the window flag bit set to 1 . Thus this mechanism increases the network life. Figure 2 explains the system architecture of the proposed work.

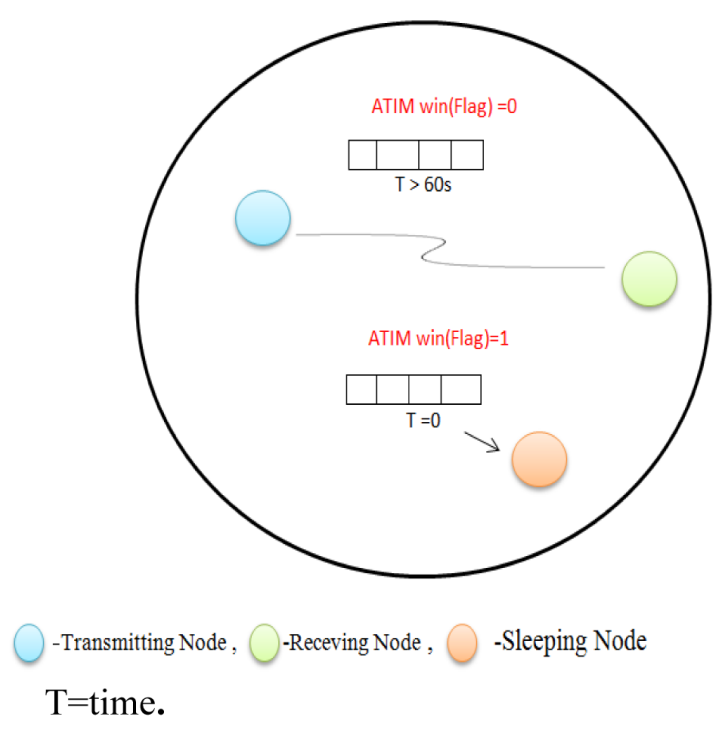

Figure 2. Network Architecture.

\section{Result}

The following metrics are used to calculate the performance of the algorithm.

Throughput: Thus the throughput calculate at the destination by measuring number of packet delivered by nodes in second.

$$
\text { Throughput }=\frac{\text { number of packet delivered }}{\text { time }}
$$

Nodes Energy: Energy consumption is calculated by number of bits delivered by the node. Nodes energy is measured by joule.

Delay: The packet delay may be calculated after receiving the ATIM acknowledgement from the receiver.

Thus, the stimulation is perform in $100 \mathrm{X} 100$ area with 10 nodes. The nodes are placed in random way point. The Figure 3 explains the node behavior with and without Fast Dormancy (FD). Figure 4 explains nodes consumed energy based on proposed algorithm.

\section{Conclusion}

This paper discuss about power saving mechanism in adhoc network. The nodes energy are wasted by overhearing in the network traffic. The $3 \mathrm{G}$ fast dormancy mechanism release the channel using ATIM window. The ATIM window used to transmit the packet between source and 


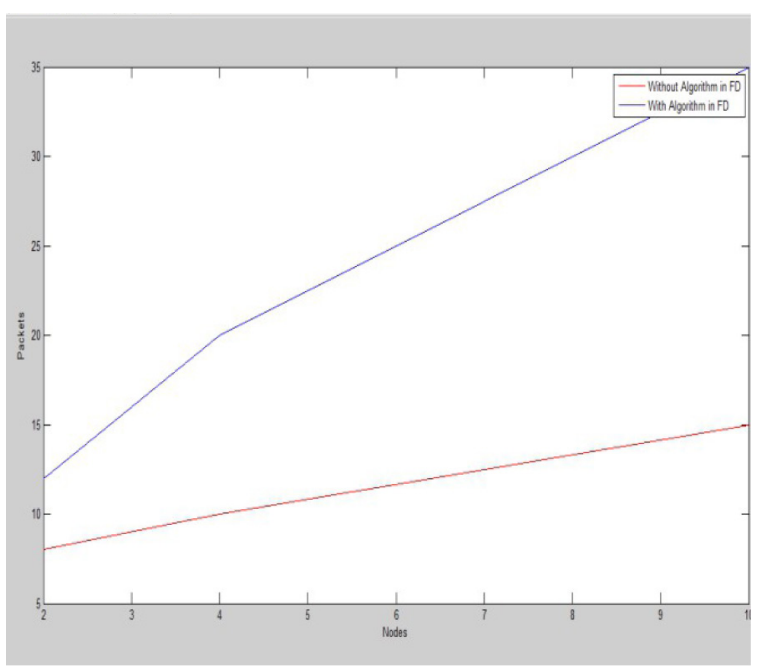

Figure 3. 10 Nodes Throughput.

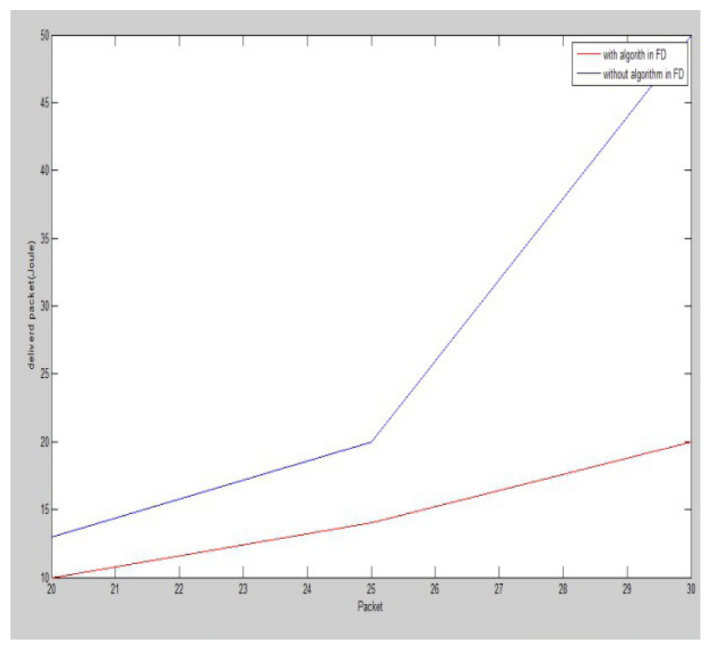

Figure 4. Nodes Energy. destination. The size of the ATIM window is defined as optimal size. Thus the ATIM window makes the node to sleep when there is no traffic and awake the node when there is a transmission. The $90 \%$ of the node alive in the network to uphold network topology.

Thus maintaining energy of the node $90 \%$ alive in the network.

\section{References}

1. Available from: http://3gnetworksbus237.blogspot. in/2011/04/advantages-and-disadvantages-of-3g.html.

2. Helen D, Arivazhagan D. Refining an Ad-hoc Network Performance by Executing Multiple Channel in Mac Protocol. IJCSIT. 2014; 5(4):5458-60.

3. Available from: http://living-smartly.com/2011/08/3gadvantages-drawbacks/.

4. Available from: http://hindisms.org/mobile-masti/how-toactivate-3g-in-tata-docomo-prepaid-faq.html

5. 3GPP Release 8:3GPP TS 25.331,2008.

6. Available from: http://www.gsmarena.com/Samsunggoogle-nexus-s-3620.php.

7. The editors of IEEE 802.11, Wireless LAN Medium Access Control (MAC) and Physical Layer (PHY) Specification.

8. Woesner H, Ebert J-P, Schlager M, Wolisz A. Power-Saving Mechanisms in Emerging Standards for Wireless LANs: The MAC Level Perspective. IEEE Personal Communications.

9. Helen D, Arivazhagan D. Applications, Advantages and Challenges of Ad Hoc Networks. JAIR; 2014 Jan; 2(8): 453-7. 